\section{S154 IS THERE A ROLE FOR IL-33 IN THE PATHOGENESIS OF PULMONARY ARTERIAL HYPERTENSION?}

doi:10.1136/thx.2010.150953.5

${ }^{1} \mathrm{D}$ Shao, ${ }^{2} \mathrm{~F}$ Perros, ${ }^{2} \mathrm{M}$ Humbert, ${ }^{3} \mathrm{G}$ Caramori, ${ }^{1} \mathrm{~L}$ Price, ${ }^{4} \mathrm{I}$ Addcock, ${ }^{1} \mathrm{~S}$ Wort. ${ }^{1}$ Unit of Thoracic Critical Care, National Heart \& Lung Institute, Royal Brompton, London, UK; ${ }^{2}$ Université Paris-Sud, Faculté de Médecine, Paris, France, ${ }^{3}$ Dipartimento di MedicinaClinica e Sperimentale, Centro di RicercasuAsma e BPCO, Ferrara, Italy; ${ }^{4}$ Department of Cell \& Molecular Biology, Airways Disease Section, National Heart and Lung Institute, Faculty of Medicine, Imperial College London, London, UK

Introduction and objectives IL- 33 is a $31 \mathrm{KDa}$ cytokine which is a member of the IL- 1 family. It resides in the nucleus of endothelial and epithelial cells as a chromatin-associated factor in vivo. IL-33 is thought to be released from stressed or necrotic, but not apoptotic, endothelial or epithelial cells in response to cell injury or infection, acting as an endogenous 'alarmin', to alert the immune system of cell and tissue damage. IL-33 is then able to bind to its receptor (ST2) on immune cells, thereby stimulating immunoregulatory activity through induction of NF- $\kappa \mathrm{B}$ and mitogen-activated protein kinases, as well as enhancing the production of the Th2 cytokines IL- 5 and IL-13. There is increasing evidence that IL-33 may have a protective role in terms of endothelial integrity and function. As the pathogenesis of pulmonary arterial hypertension (PAH) is thought to involve endothelial cell dysfunction, we were interested to see whether there may be a role for IL-33 in this condition.

Methods RT-PCR for IL-33 was performed on human pulmonary arterial cells (HPAECs) derived from normal healthy controls and patients with idiopathic PAH. siRNA IL-33 knockdown was performed using smartpool duplexes on normal HPAECs. RT-PCR was then performed using QuantiTec primer assays.

Results IL-33 mRNA expression was decreased 2.1-fold in PAH patient samples $(0.369 \pm 0.02, n=10)$ compared to controls $(0.761 \pm 0.06, n=14) p<0.005$. In normal human lung tissue, intense IL-33 staining was shown in the ECs nuclear in blood vessels. It is also worth to note that there was little or no IL-33 staining in nonECs. siRNA knockdown IL-33 in HPAECs resulted in a $1.8 \pm 0.22$, $1.3 \pm 0.13$ and $1.4 \pm 0.20(n=3)$ fold increase of mRNA IL-6, BMP-9 and ST2 mRNA, respectively; whereas RANTES, fractalkine and cathepsin-L mRNA was decreased by $1.5 \pm 0.03,1.7 \pm 0.01$ and $2.1 \pm 0.17$ fold respectively $(n=3)$ (Abstract S154 Figure 1).

Conclusion IL-33mayplay an important role in the pathogenesis of $\mathrm{PAH}$ through regulating the expression ofcytokines and chemokines known to be involved in vascular remodelling. In particular, it is of interest that II-33 may regulate IL- 6 production and ST2 which acts as an endogenous IL-33 inhibitor.

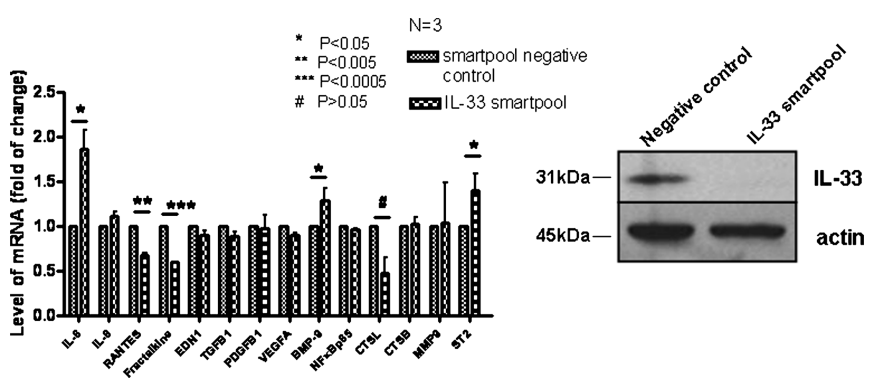

Abstract S154 Figure 1 Effect of IL-33 on gene expression.

\section{S155 THE ROLE OF ST2 IN A MODEL OF PULMONARY HYPERTENSION}

doi:10.1136/thx.2010.150953.6

${ }^{1}$ A Mahmood, ${ }^{2} \mathrm{C}$ McSharry, ${ }^{2} \mathrm{D}$ Xu, ${ }^{1} \mathrm{~A} J$ Peacock, ${ }^{1} \mathrm{D} \mathrm{J}$ Welsh. ${ }^{1}$ Scottish Pulmonary Vascular Unit, Western Infirmary, Glasgow, UK; ${ }^{2}$ Dept of Immunology, University of Glasgow, Glasgow, UK

Background Pulmonary arterial hypertension (PAH) is a fatal condition involving remodelling of the pulmonary vessel wall leading to elevated pulmonary arterial pressure and eventually right heart failure. ST2 is a transmembrane receptor with ligand interleukin-33 (IL-33). p38 MAP kinase is an intracellular signalling molecule shown to be involved in the vascular remodelling associated with PAH; and is also involved in ST2/IL-33 signalling. ST2/ IL-33 signalling has been shown to reduce fibrosis in the heart following pressure overload in animal models.

Aims To determine whether ST2/IL-33 signalling was involved in the proliferation of mouse pulmonary artery fibroblasts and if p38 MAP kinase was involved.

Methods Two cell types were used-wild type (WT) and ST2 knockout (ST2-/-) mouse pulmonary artery fibroblasts. Proliferation was assessed by $\left[{ }^{3} \mathrm{H}\right]$ Thymidine incorporation. Expression of p38 MAP kinase was detected by Western blotting. Cells were cultured at various serum concentrations in normoxia and hypoxia. A p38 MAP kinase inhibitor (SB203580) was used to assess its role in cell proliferation. The effect of IL-33 on cell proliferation and expression of p38 MAP kinase was studied.

Results WT and ST2-/- cells proliferated in response to increasing serum concentration. WT cells hyperproliferated in response to hypoxia. ST2-/ - cells hyperproliferated in normoxia and hypoxia compared to WT cells. The hyperproliferation of ST2-/- cells in normoxia and hypoxia could be reduced by p38 MAP kinase inhibition (p38) (see Abstract S155 Figure 1). Phosphorylated p38 MAP kinase was detected in all ST2-/- cells and in WT cells in hypoxia with $10 \%$ serum, demonstrating p38 MAP kinase is involved in the

ST2-/- Mouse Pulmonary Artery Fibroblasts in Normoxia with p38 MAP Kinase Inhibitor

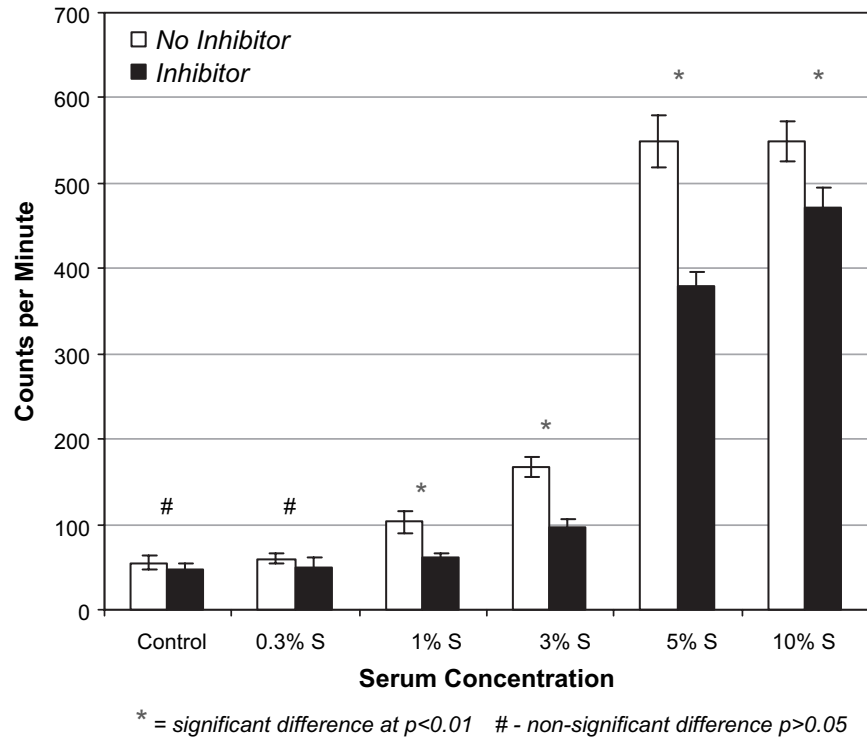

Abstract S155 Figure 1 Proliferation in Response to pp38 MAP Kinase Inhibition in Normoxia ST2-/- cells were cultured in normoxia at serum concentrations $0,0.3,1,3,5$ and $10 \%$, with and without the p38 MAP kinase inhibitor SB203580. Proliferation was reduced in the presence of the inhibitor with statistical significance at serum concentrations $1 \%$ serum suggesting that p38 MAP kinase is involved in the hyperproliferation seen in ST2-/- cells. 